\section{Federation University ResearchOnline}

\section{https://researchonline.federation.edu.au}

Copyright Notice

This is the peer-reviewed version of the following article:

Miloyan, \& Pachana, N. A. (2016). Clinical Significance of Individual GAD Symptoms in Later Life. Journal of Geriatric Psychiatry and Neurology, 29(2), 92-98.

The online version of this article can be found at:

https://doi.org/10.1177/0891988715606231

Copyright ( 2015 SAGE Publications who receive access to an article through a repository are reminded that the article is protected by copyright. Users may download and save a local copy of an article accessed in an institutional repository for the user's personal reference. For permission to reuse an article, please follow the SAGE Process for Requesting Permission. 


\title{
Clinical Significance of Individual GAD Symptoms in Later Life
}

\author{
Beyon Miloyan, PhD $^{1}$ and Nancy A. Pachana, PhD $^{2}$ \\ ${ }^{1}$ Department of Mental Health, Johns Hopkins Bloomberg School of Public Health, Baltimore, MD, \\ USA \\ ${ }^{2}$ School of Psychology, The University of Queensland, Brisbane, Australia
}

\begin{abstract}
Background-There are age-related differences in the manifestation of generalized anxiety disorder (GAD) symptoms and their associated impact on psychosocial and functional status. However, it remains unclear whether specific symptoms (or symptom patterns) are of comparable clinical significance across different functional domains.

Methods-A sample of 865 self-reported worriers (aged 60 years and older) who endorsed GAD screening questions in the Alcohol Use Disorder and Associated Disabilities Interview Schedule, Diagnostic and Statistical Manual of Mental Disorders, fourth edition, were derived from Wave 1 of the National Epidemiological Survey of Alcohol and Related Conditions.
\end{abstract}

Results-Being easily fatigued was significantly associated with occupational disability and a functional disability variable (restricting usual activity in any way). Irritability was significantly associated with social friction (arguments with friends, family, or colleagues) and a functional disability variable (found was unable to do something wanted to do), and poor self-perceived health was also associated with the same functional disability variable. Excessive worry, despite being among the least reported symptoms, was significantly associated with distress.

Conclusions-These findings suggest that individual GAD symptoms are differentially associated with aspects of clinical significance relevant to daily life, such as social, occupational, and functional ability. The differential impact of individual symptoms on functional status may be diluted when using symptom sum scores. A nuanced approach to assessing the clinical significance of individual GAD symptoms in older adults may be fruitful for efforts aimed at early detection and treatment.

\section{Keywords}

anxiety; functional status; geriatric psychiatry

There are age-related differences in the presentation of generalized anxiety disorder (GAD) that may have a particular bearing on diagnosis and treatment in older adults. A diagnosis of

Reprints and permission: sagepub.com/journalsPermissions.nav

Corresponding Author: Beyon Miloyan, Johns Hopkins Center on Aging and Health, 2024 E. Monument, Street, Suite 2-700, Baltimore, MD 21205, USA. beyon@jhu.edu.

Declaration of Conflicting Interests

The author(s) declared no potential conflicts of interest with respect to the research, authorship, and/or publication of this article. 
GAD requires that its constituent symptoms be clinically significant (ie, associated with considerable distress and/or disability). In terms of GAD symptom patterns, relative to younger adults, older adults tend to report fewer worries, ascribe less functional value to worrying, utilize fewer coping strategies in an attempt to control their worries, and worry more about others than themselves. ${ }^{1-6}$ In terms of clinical significance, older adults are more vulnerable to physical disability and less vulnerable to emotional distress and negative affective states, suggesting that there may be age-related variables influencing the functional impact of GAD symptoms (ie, fewer symptoms may suffice to cause disability in older adults). ${ }^{7-9}$ Indeed, fewer symptoms have been found to distinguish older adults with threshold and subthreshold GAD, older adults with threshold and subthreshold GAD have been found to share similarities in terms of health-related characteristics, and anxietyassociated disability observed in older adults with GAD has been found to persist longitudinally despite the waning of symptoms. ${ }^{10-12}$ However, most studies assessing the impact of GAD symptoms have to date relied on symptom sum scores, and the influence of individual GAD symptoms on clinical significance variables therefore remains unclear. The purpose of this study was to determine whether aspects of clinical significance associated with late-life GAD would be accounted for on the basis of unique symptom patterns.

\section{Methods}

\section{Sample}

The National Epidemiologic Survey of Alcohol and Related Conditions (NESARC) was conducted in 2001 to 2002 by the US Bureau of the Census and sponsored by the National Institute of Alcohol Abuse and Alcoholism (NIAAA). Forty-three thousand ninety-three adults were sampled from all 50 US States, and the District of Columbia. African Americans, Hispanics, and young adults were purposively oversampled, and the overall response rate was $81 \%$. Data were adjusted for oversampling and nonresponse. Face-to-face interviews were conducted by trained lay interviewers using the Alcohol Use Disorder and Associated Disabilities Interview Schedule, Diagnostic and Statistical Manual of Mental Disorders, fourth edition, version (AUDADIS-IV, DSM-IV), which has been found to have good psychometric properties. ${ }^{13,14}$ Specifically, GAD symptoms were found to have very good reliability, and the GAD diagnostic variable was found to be fairly reliable. ${ }^{13}$ All participants aged 60 years and older who endorsed at least 1 of the 2 screening questions for the GAD portion of the interview were included in this study because those who did not endorse the screening questions were not further assessed for the GAD portion of the interview. The 2 screening questions were: "Ever had a 6+ month period during which you felt: (a) tense/nervous/worried most of the time or (b) very tense/ nervous/worried most of the time about everyday problems?" The overall sample consisted of 865 participants (aged 60-98 years), representing approximately one-fourth of the overall sample of self-reported worriers. In other words, about $25 \%$ of all self-reported worriers in the overall NESARC sample were aged 60 years or older.

\section{Measures}

Sociodemographic and health variables were accounted for in all analyses. These consisted of gender, education (some college or higher, completed high school, less than high school), 
personal income (US \$0-US \$9999, US \$10 000-US \$34 999, US \$35 000+), marital status (married/cohabiting, widowed/divorced/separated, never married), urbanicity (urban, rural), self-perceived health (excellent, very good, good, fair, poor), and presence of chronic medical conditions $(0,1$, or 2 or more; selected from a finite list of 11 variables including arteriosclerosis, hypertension, liver cirrhosis, liver disease, angina pectoris, tachycardia, myocardial infarction, heart disease, stomach ulcer, gastritis, or arthritis). Participants were also characterized with respect to current and lifetime anxiety disorders (not including GAD), current and lifetime mood disorders, and personality disorders, all based on the AUDADIS-IV. The participants in this study, who endorsed at least 1 of the 2 GAD screening questions, could be conceptualized as having 12-month threshold or subthreshold GAD (consistent with previous studies). ${ }^{11,12}$ The sociodemographic and health variables of this sample are summarized in Table 1.

The following 10 core GAD symptoms included were excessive worry (ever think your worrying was excessive?), difficulty controlling worry (ever find it difficult to stop being tense/nervous/worried?), restlessness (often became so restless you fidgeted, often paced, or couldn't sit still), feeling keyed up or on edge (often felt keyed up or on edge), being easily fatigued (often got tired easily), difficulty concentrating (often had trouble concentrating/ keeping mind on things), mind going blank (often had times when forgot what talking about/ mind went blank), irritability (often felt irritable), muscle tension (often had tense, sore, or aching muscles), and sleep disturbance (often had trouble falling/staying asleep). All symptoms were obtained from the GAD portion of the AUDADIS-IV. Participants who met symptom criteria and reported occurrence in the past year were given a 12-month DSM-IV GAD diagnosis.

Five self-reported symptoms relevant to the clinical significance criterion, derived from the same portion of the interview, were also included: distress (felt uncomfortable about feeling nervous/anxious or by any of those things going on at the same time), social friction (had arguments/friction with family, friends, people at work, or anyone else), occupational disability (had difficulty doing things supposed to do-working, schoolwork, taking care of house/family), and 2 functional impairment variables (A: restricted usual activity in any way; B: found was unable to do something wanted to do).

\section{Statistical Analyses}

Five sets of weighted binary logistic regression analyses were conducted, in which each of the clinical significance variables served as the outcome variable of interest. Predictor variables included each of the sociodemographic, health, and symptom variables. An additional weighted binary logistic regression analysis was conducted, in which 12-month GAD diagnostic status (threshold or subthreshold) was the outcome variable, and predictor variables included sociodemographic, health, comorbidity, and clinical significance variables. Due to the inclusion of a large number of variables, significance levels were reset to $P<.01$ in each of the analyses to account for type 1 error. Sampling weights were used to adjust for oversampling and nonresponse and were based on census data. All statistical analyses were conducted using Stata 12.0, and survey commands consisted of primary 
sampling units, probability weights, and strata with only 1 sampling unit centered at the overall sample mean (rather than the stratum mean).

\section{Results}

\section{Individual GAD Symptom Frequencies}

Figure 1 displays the weighted proportion (and 95\% confidence intervals [CIs]) of participants endorsing each symptom. The 5 most frequently reported symptoms, with affirmation from $50 \%$ or more of all self-reported worriers, were difficulty controlling worry (0.69; 95\% CI: 0.65-0.72), being easily fatigued (0.61; 95\% CI: 0.56-0.65), feeling keyed up or on edge $(0.54 ; 95 \%$ CI: $0.50-0.58)$, experiencing sleep disturbance $(0.52 ; 95 \% \mathrm{CI}$ : $0.48-0.56)$, and difficulty concentrating $(0.51 ; 95 \% \mathrm{CI}: 0.47-0.55)$. Interestingly, one of the key symptoms for meeting GAD criteria, excessive worry $(0.33$; 95\% CI: $0.29-0.37)$, was among the least reported symptoms.

\section{Individual Clinical Significance Variable Frequencies}

Figure 2 displays the weighted proportion (and 95\% CIs) of participants endorsing each functional impact variable. About half of all self-reported worriers reported being distressed $(0.51 ; 95 \% \mathrm{CI}: 0.46-0.56)$ and about a quarter of all participants reported experiencing social friction $(0.30 ; 95 \% \mathrm{CI}: 0.25-0.35)$.

\section{Symptoms Associated With Clinical Significance Variables}

In general, different individual symptoms were found to be associated with different functional impact variables (See Table 2). Excessive worry was significantly associated with distress (odds ratio [OR]: 2.12; 95\% CI: 1.31-3.42). Irritability was significantly associated with social friction (OR: 3.38; 95\% CI: 2.00-5.71). Being easily fatigued was significantly associated with occupational disability (OR: 2.50; 95\% CI: 1.40-4.47) and functional disability (restricted usual activity in any way; OR: 2.43; 95\% CI: 1.34-4.41). Irritability was significantly associated with another functional disability variable (found was unable to do something wanted to do; OR: 1.87 ; 95\% CI: 1.20-2.92).

\section{Clinical Significance Variables Associated With Diagnostic Status}

An additional binary logistic regression analysis was conducted in order to assess whether any clinical significance variables would distinguish those with threshold and subthreshold GAD (see Table 3). Only a concurrent 12-month diagnosis of any mood disorder was found to significantly distinguish both groups (OR: 6.61; 95\% CI: 2.96-14.77), such that a higher proportion of those with GAD had a comorbid mood disorder.

\section{Discussion}

In this study, proportions of old-aged self-reported worriers endorsing each of the 10 symptoms typically associated with a DSM-IV GAD diagnosis were estimated. Intriguingly, 1 of the 3 core GAD symptoms - excessive worry - was among the least reported symptoms. However, difficulty controlling worry was endorsed by the highest proportion of participants. These findings are consistent with those of previous studies, reporting that 
uncontrollability, but not excessiveness of worry, may better represent a core feature of GAD. ${ }^{15,16}$ The third core symptom of GAD (ie, 6 or more months of worry on more days than not) was not included in these analyses because this question was used to determine the present sample (owing to a design feature of the NESARC). Being easily fatigued, feeling keyed up or on edge, experiencing sleep disturbance, and difficulty concentrating were among the most highly reported symptoms, with affirmation from at least half of all participants. These symptoms may be due to or exacerbated by normal aging, cognitive decline, or comorbid physical and psychiatric conditions (ie, depression or cognitive impairment). Given the lack of an asymptomatic comparison group, and the binary responses required for these variables, the present study was unable to determine the extent to which these symptoms are exacerbated by a GAD diagnosis.

Next, the proportion of old-aged self-reported worriers who endorsed each of the 5 functional impact variables was estimated. Distress and 1 of the 2 functional disability variables were found to be the most extensively reported, with affirmation from nearly half of all participants. Occupational disability and social friction were the least frequently reported. At first glance, the finding that distress was the most highly rated of all functional impact variables appears to be inconsistent with previous findings, suggesting that emotional experience improves with age. ${ }^{8}$ However, given that this study exclusively included participants who reported that they had experienced at least 6 months of worry, it would perhaps be more surprising if distress was not so highly rated. Although older adults are generally less prone to experiencing negative affective states, due in large part to improved emotional regulation, there are within-group individual differences such that some older adults remain susceptible to experiencing persistent and chronic negative states (eg, worry and anxiety). ${ }^{17}$

Finally, this study aimed to determine whether specific symptoms, or symptom patterns, would consistently be associated with various clinical significance variables. We hypothesized that different symptoms (or symptom clusters) would account for different clinical significance variables. In general, the findings of this study supported our hypothesis. A different pattern of symptoms was associated with each functional impact variable, the only exceptions being occupational disability and a functional disability variable (restricting usual activity in any way), which were accounted for by "being easily fatigued." Despite the observation that excessive worry was found to be among the least frequently reported symptoms by this sample, it was the only variable that was significantly associated with distress. These findings are somewhat consistent with those of a previous study, which reported that participants who did not meet the excessiveness criterion for GAD had a slightly less extreme manifestation of anxiety (despite comparability on a number of other variables). ${ }^{15}$ However, at least among the older adults in this sample, excessive worry was significantly associated with distress despite being infrequently reported.

These findings are also consistent with previous studies, which indicate that individual symptoms, and symptom patterns, associated with late-life GAD (and with psychiatric disorders in general) have differential influences on diagnostic outcomes. ${ }^{12}$ Individual symptoms appear to have different risk factors that may be diluted using sum scores. ${ }^{18}$ For 
example, a recent study on depressed individuals found that individual symptoms have differential impacts on psychosocial outcomes. ${ }^{19}$ Focusing on aspects of psychosocial outcomes associated with individual symptoms may be a fruitful approach for improving conceptualization, identification, and treatment of GAD in later life as well. Our findings suggest that specific physiological (eg, being easily fatigued) and emotional (eg, irritability) symptoms, in addition to worry, are associated with specific functional status variables among those with threshold and subthreshold GAD. Like depression, GAD could be conceptualized as being composed of symptom clusters that may be a cause or consequence of each other. ${ }^{20}$ Older age appears to bring about some regular changes in the manifestation of these symptoms, such that physical symptoms become more highly prevalent in the context of GAD. ${ }^{5}$ However, these changes may be due to a mix of age effects and comorbid physical and psychiatric conditions, and the interactions between these symptoms are less well known. Specifically, more work is needed to understand the interplay between physical symptoms and worry in later life and the mechanisms by which physical symptoms may exacerbate GAD outcomes. Nonetheless, these findings cast doubt on solely worry-based assessment for detecting GAD and the efficacy of cognitive interventions for treating latelife GAD (which have been found to be at best moderately effective). ${ }^{21-23}$

Interestingly, there were no significant differences between the proportion of older adults with threshold and subthreshold GAD endorsing any of the clinical significance variables. These findings are consistent with previous studies indicating that there are relatively marginal differences between older adults with threshold and subthreshold GAD. ${ }^{10-12}$ However, it is important to note that clinical significance variables required binary responses (yes or no). Therefore, it was not possible to determine whether there were differences in the severity of distress or specific disability variables with respect to diagnostic status. Relative to the subthreshold group in this study, however, a significantly higher proportion of those with a diagnosis of GAD met criteria for a concurrent mood disorder. There are additional limitations with the present study that must be accounted for. As noted, the design of the NESARC prevented any comparison between self-reported worriers and an asymptomatic group. Thus, it was not possible to completely disentangle normal age effects from the unique influence of anxiety on symptom presentation and associated clinical significance variables. Second, the findings are based on cross-sectional associations, and therefore, causality could not be determined. Third, responses to all variables were obtained through self-report. Although self-report is effective for the identification of subjective symptoms, informant report is more effective for identifying the severity of observable symptoms. ${ }^{24}$ Despite these limitations, we believe this study provides an important first step toward understanding the functional impact of individual GAD symptoms in later life. Future studies investigating longitudinal changes in symptom patterns and their functional impact will play an important role in improving conceptualization of late-life GAD.

\section{Acknowledgments}

\section{Funding}

The author(s) disclosed receipt of the following financial support for the research, authorship, and/or publication of this article: This work was supported by the Johns Hopkins Epidemiology and Biostatistics of Aging Training Program of the National Institute on Aging (NIA) under award number T32AG000247. 


\section{References}

1. Basevitz P, Pushkar D, Chaikelson J, Conway M, Dalton C. Age-related differences in worry and related processes. Int J Aging Hum Dev. 2008; 66(4):283-305. [PubMed: 18507331]

2. Hunt S, Wisocki P, Yanko J. Worry and use of coping strategies among older and younger adults. J Anxiety Disord. 2003; 17(5):547-560. [PubMed: 12941365]

3. Gonçalves DC, Byrne GJ. Who worries most? Worry prevalence and patterns across the lifespan. Int J Geriatr Psychiatry. 2013; 28(1):41-49. [PubMed: 22374912]

4. Gould CE, Edelstein BA. Worry, emotion control, and anxiety control in older and younger adults. J Anxiety Disord. 2010; 24(7):759-766. [PubMed: 20708492]

5. Miloyan B, Pachana NA. Clinical significance of worry and physical symptoms in late-life GAD. Int J Geriatr Psychiatry. 2015 [published online February 20, 2015].

6. Wetherell JL, Le Roux H, Gatz M. DSM-IV criteria for generalized anxiety disorder in older adults: distinguishing the worried from the well. Psych Aging. 2003; 18(3):622-627.

7. Brault MW. Americans with disabilities: 2010. Household Economic Studies. Current Population Reports. 2012:70-131.

8. Carstensen LL, Turan B, Scheibe S, et al. Emotional experience improves with age: evidence based on over 10 years of experience sampling. Psych Aging. 2011; 26(1):21-33.

9. Lenze EJ, Rogers JC, Martire LM, et al. The association of late-life depression and anxiety with physical disability. Am J Geriatr Psychiatry. 2001; 9(2):113-135. [PubMed: 11316616]

10. Grenier S, Préville M, Boyer R, et al. the Scientific Committee of the ESA Study. The impact of the DSM-IV symptom and clinical significance criteria on the prevalence estimates of subthreshold and threshold anxiety in the older adult population. Am J Geriatr Psychiatry. 2011; 19(4):316-326. [PubMed: 21427640]

11. Miloyan B, Byrne GJ, Pachana NA. Age-related changes in generalized anxiety disorder symptoms. Int Psychogeriatr. 2014; 26(4):565-572. [PubMed: 24405581]

12. Miloyan B, Byrne GJ, Pachana NA. Threshold and subthreshold generalized anxiety disorder in later life. Am J Geriatr Psychiatry. 2015; 23(6):633-641. [PubMed: 25240936]

13. Grant BF, Dawson DA, Stinson FS, Chou PS, Kay W, Pickering R. The alcohol use disorder and associated disabilities interview schedule-IV (AUDADIS-IV): reliability of alcohol consumption, tobacco use, family history of depression and psychiatric diagnostic modules in a general population sample. Drug Alcohol Depend. 2003; 71(1):7-16. [PubMed: 12821201]

14. Ruan WJ, Goldstein RB, Chou P, et al. The alcohol use disorder and associated disabilities interview schedule-IV (AUDADIS-IV): reliability of new psychiatric diagnostic modules and risk factors in a general population sample. Drug Alcohol Depend. 2008; 92(1-3):27-36. [PubMed: 17706375]

15. Ruscio AM, Lane M, Roy-Byrne P, et al. Should excessive worry be required for a diagnosis of generalized anxiety disorder? Results from the US National Comorbidity Survey Replication. Psychol Med. 2005; 35(12):1761-1772. [PubMed: 16300690]

16. Hallion LS, Ruscio AM. Should uncontrollable worry be removed from the definition of GAD? A test of incremental validity. J Abnorm Psychol. 2013; 122(2):369-375. [PubMed: 23713499]

17. Charles ST, Luong G. Emotional experience across adulthood: the theoretical model of strength and vulnerability integration. Curr Dir Psychol Sci. 2013; 22(6):443-448.

18. Fried EI, Nesse RM, Zivin K, Guille C, Sen S. Depression is more than the sum core of its parts: individual DSM symptoms have different risk factors. Psychol Med. 2014; 44(10):2067-2076. [PubMed: 24289852]

19. Fried EI, Nesse RM. The impact of individual depressive symptoms on impairment of psychosocial functioning. PLoS One. 2014; 9(2):e90311. [PubMed: 24587318]

20. Fried EI. Problematic assumptions have slowed down depression research: why symptoms, not syndromes are the way forward. Front Psychol. 2015; 6:309. [PubMed: 25852621]

21. Bryant C, Mohlman J, Gum A, et al. Anxiety disorders in older adults: looking to DSM5 and beyond. Am J Geriatr Psychiatry. 2013; 21(9):872-876. [PubMed: 23567396] 
22. Gonçalves DC, Byrne GJ. Interventions for generalized anxiety disorder in older adults: systematic review and meta-analysis. J Anxiety Disord. 2012; 26(1):1-11. [PubMed: 21907538]

23. Thorp SR, Ayers CR, Nuevo R, Stoddard JA, Sorrell JT, Wetherell JL. Meta-analysis comparing different behavioural treatments for late-life anxiety. Am J Geriatr Psychiatry. 2009; 17(2):105115. [PubMed: 19155744]

24. McDade-Montez EA, Watson D, O'Hara MW, Denburg NL. The effect of symptom visibility on informant reporting. Psych Aging. 2008; 23(4):940-946. 
Difficulty Controlling Worry

Easily Fatigued

Keyed Up or On Edge

Sleep Disturbance

Difficulty Concentrating

Irritability

Mind Going Blank

Muscle Tension

Excessive Worry

Restless
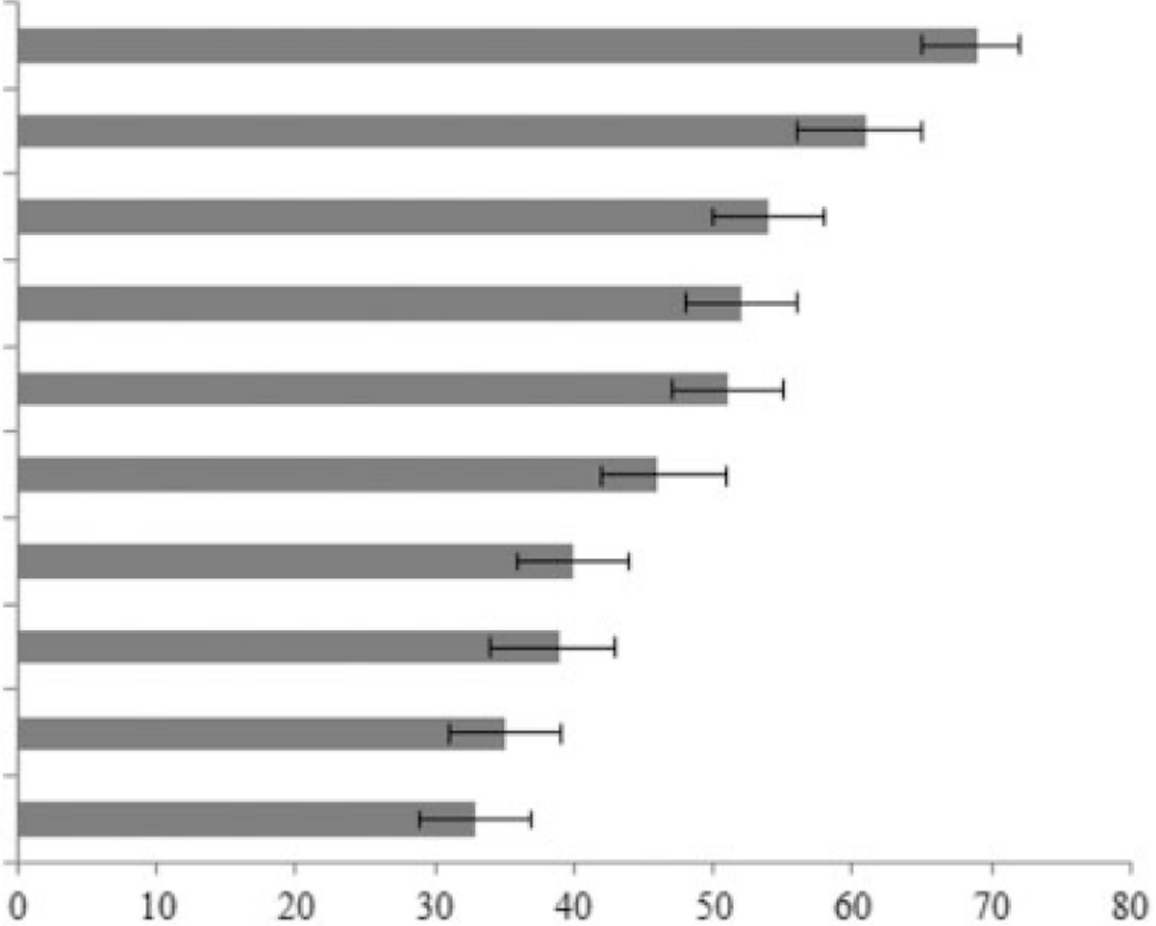

Proportion

Figure 1.

Weighted proportion (and 95\% confidence interval [CI] bars) of core generalized anxiety disorder (GAD) symptoms endorsed by self-reported worriers. 
Functional Disability B

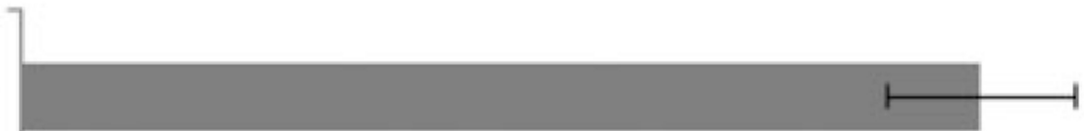

Functional Disability A

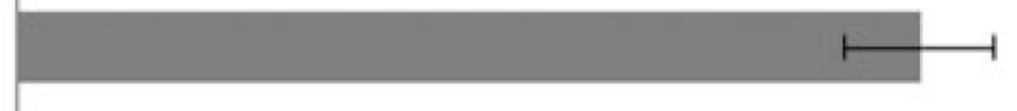

Distress

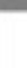

Occupational Disability

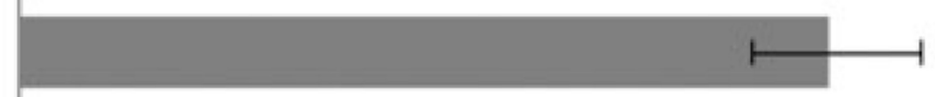

Social Friction

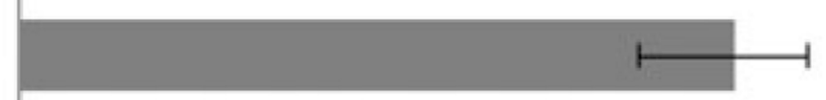

Figure 2.

Weighted proportion (and 95\% confidence interval [CI] error bars) of clinical significance variables endorsed by self-reported worriers. Functional disability A refers to participants reporting that their usual activities were restricted, and functional disability B refers to participants reporting that they were unable to do something that they wanted to do. 
Table 1

Sociodemographic Characteristics of Self-Reported Worriers Aged 60 Years and Older. ${ }^{a}$

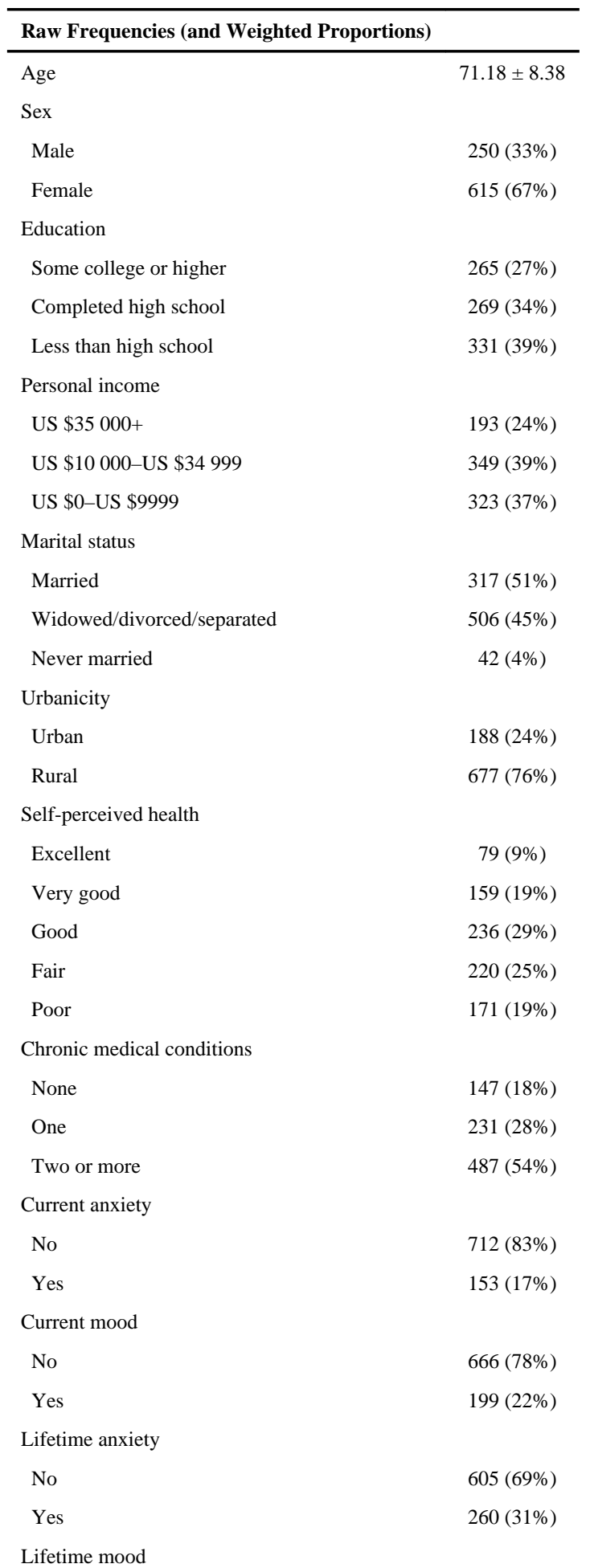




\begin{tabular}{ll}
\hline Raw Frequencies (and Weighted Proportions) & \\
\hline No & $451(53 \%)$ \\
Yes & $414(47 \%)$ \\
Lifetime personality & \\
No & $661(77 \%)$ \\
Yes & $204(23 \%)$ \\
\hline
\end{tabular}

${ }^{a}$ Weighted percentages are rounded and do not always add to 100 . 


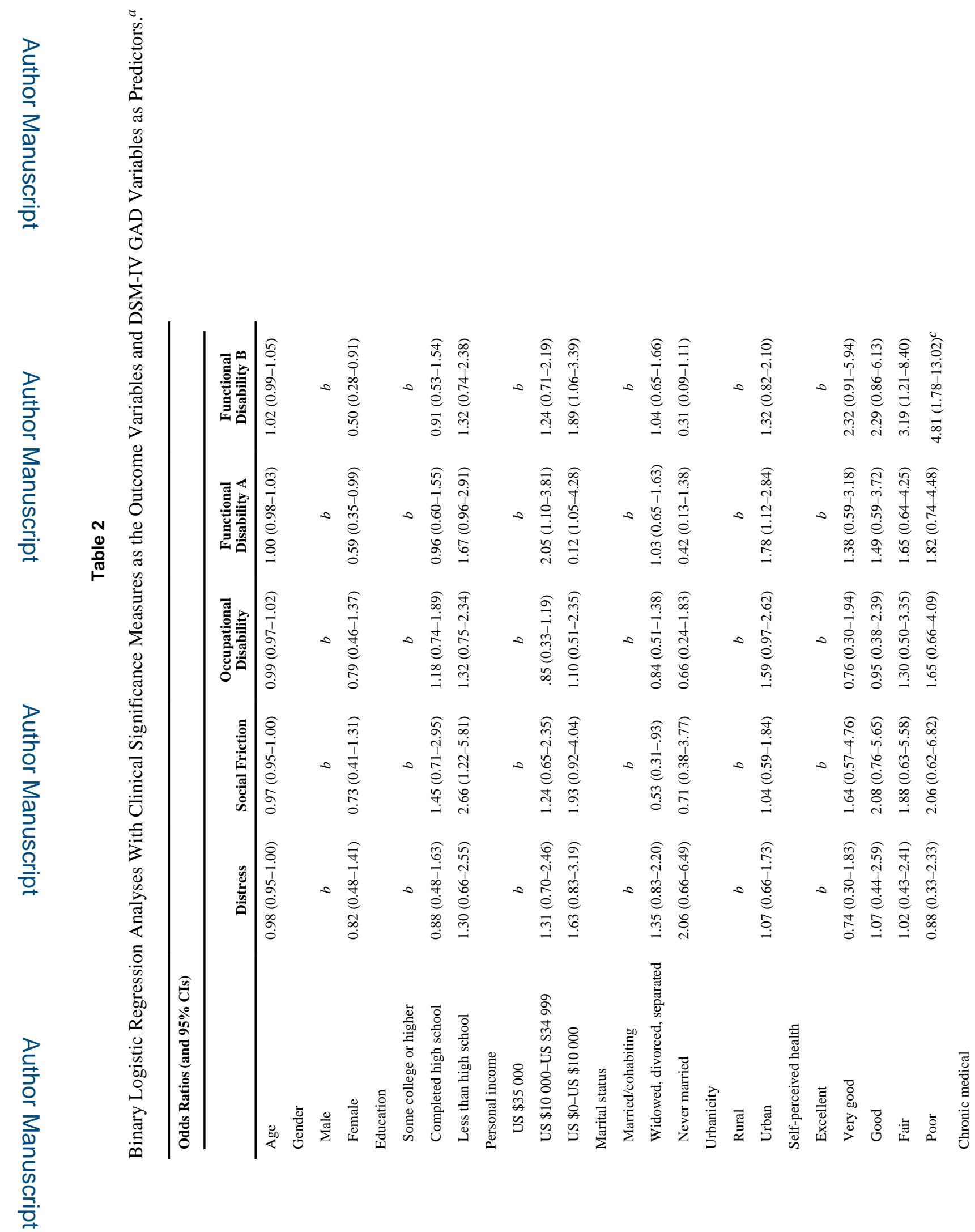

J Geriatr Psychiatry Neurol. Author manuscript; available in PMC 2017 March 01. 


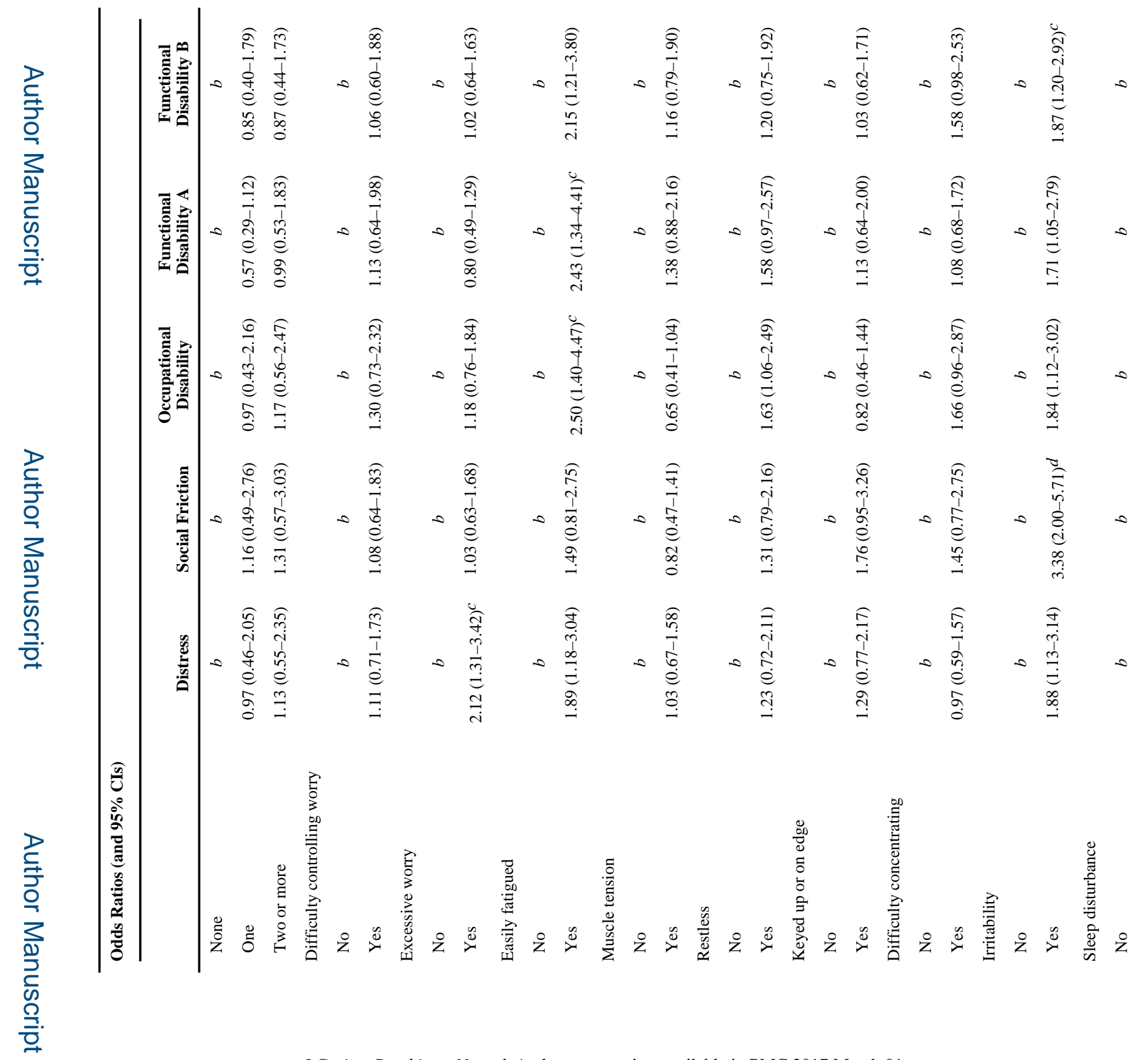




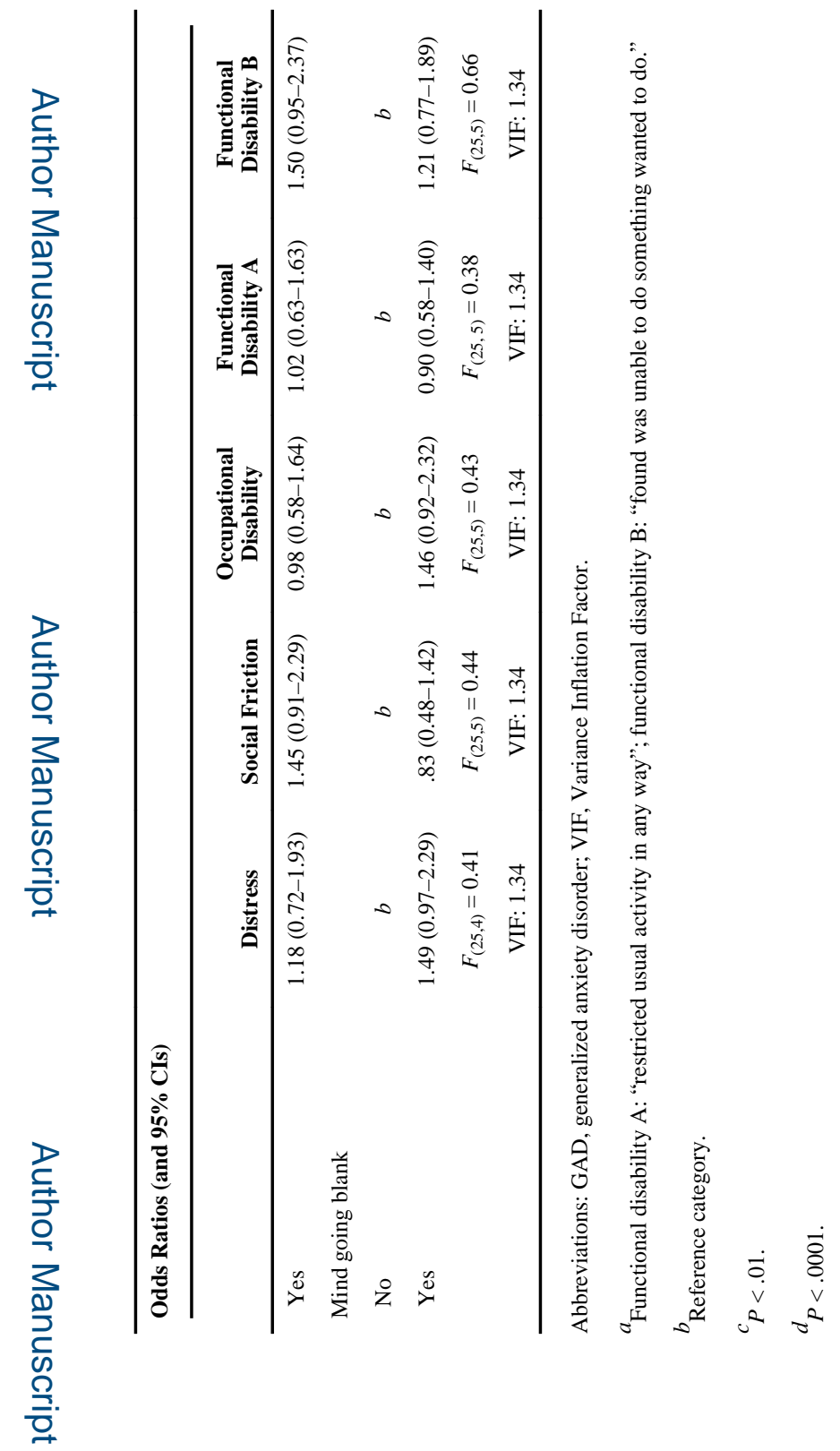

J Geriatr Psychiatry Neurol. Author manuscript; available in PMC 2017 March 01. 


\section{Table 3}

Binary Logistic Regression Distinguishing Subthreshold and Threshold GAD With Clinical Significance Variables as Predictors. ${ }^{a, b}$

\begin{tabular}{|c|c|}
\hline Age & $0.99(0.96-1.03)$ \\
\hline \multicolumn{2}{|l|}{ Gender } \\
\hline Male & $c$ \\
\hline Female & $1.98(0.97-4.06)$ \\
\hline \multicolumn{2}{|l|}{ Education } \\
\hline Some college or higher & $c$ \\
\hline Completed high school & $1.15(0.60-2.18)$ \\
\hline Less than high school & $1.19(0.65-2.17)$ \\
\hline \multicolumn{2}{|l|}{ Personal income } \\
\hline USS $\$ 35000$ & $c$ \\
\hline US \$10 000-US \$34 999 & $0.78(0.36-1.71)$ \\
\hline US $\$ 0-$ US $\$ 10000$ & $1.84(0.87-3.90)$ \\
\hline \multicolumn{2}{|l|}{ Marital status } \\
\hline Married/cohabiting & $c$ \\
\hline Widowed, divorced, separated & $1.09(0.60-2.00)$ \\
\hline Never married & $1.80(0.45-7.29)$ \\
\hline \multicolumn{2}{|l|}{ Urbanicity } \\
\hline Rural & $c$ \\
\hline Urban & $1.21(0.68-2.16)$ \\
\hline \multicolumn{2}{|l|}{ Self-perceived health } \\
\hline Excellent & $c$ \\
\hline Very good & $1.55(0.35-6.86)$ \\
\hline Good & $1.74(0.43-7.13)$ \\
\hline Fair & $1.92(0.46-8.02)$ \\
\hline Poor & $2.42(0.60-9.73)$ \\
\hline \multicolumn{2}{|l|}{ Chronic medical conditions } \\
\hline None & $c$ \\
\hline One & $0.84(0.30-2.32)$ \\
\hline Two or more & $0.83(0.33-2.05)$ \\
\hline \multicolumn{2}{|l|}{ Current anxiety disorder } \\
\hline No & $c$ \\
\hline Yes & $1.73(0.68-4.37)$ \\
\hline \multicolumn{2}{|l|}{ Current mood disorder } \\
\hline No & $c$ \\
\hline Yes & $6.61(2.96-14.77)^{d}$ \\
\hline \multicolumn{2}{|l|}{ Lifetime anxiety disorder } \\
\hline No & $c$ \\
\hline Yes & $0.73(0.29-1.83)$ \\
\hline
\end{tabular}


No

Yes

Lifetime personality disorder

No

Yes

Distress

No

Yes

Social friction

No

Yes

Occupational disability

$$
\text { No }
$$

Yes

Functional disability A

No

Yes

Functional disability B

No

Yes c

$0.49(0.22-1.09)$

$1.32(0.71-2.44)$

$c$

$1.62(0.87-3.04)$

$c$

$0.96(0.50-1.81)$

$c$

$1.20(0.60-2.40)$

$c$

$1.20(0.60-2.40)$

$c$

$1.79(0.89-3.60)$

$F_{(25,6)}=0.63$

VIF: 1.30

Abbreviations: GAD, generalized anxiety disorder; VIF, Variance Inflation Factor.

${ }^{a}$ Values are presented as odds ratios (and 95\% confidence intervals).

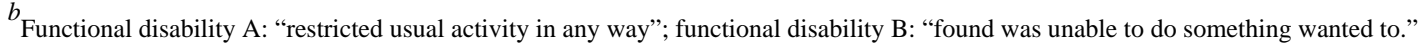

$c_{\text {Reference category. }}$

$d_{P<.0001 .}$ 